\title{
Editorial
}

\section{Progress in the Battle Against Nosocomial Legionnaires' Disease: Shedding Light on Shades of Gray}

\author{
Donald E. Craven, MD
}

\section{Remember how much you don't know.- William Osler, circa 1900 \\ Remember how much you know, but haven't applied.- Osler's ghost, 2003}

In 1977, Fraser et al. described an outbreak of pneumonia among legionnaires attending a convention at a hotel in Philadelphia in $1976 .{ }^{1}$ The outbreak, which later became known as legionnaires' disease, was caused by a new pleomorphic, faintly staining gram-negative bacillus, Legionella pneumophila, that was isolated at the Center for Disease Control from lung tissues of legionnaires who died. ${ }^{2}$ Legionella are a fascinating group of intracellular pathogens that often inhabit aquatic environments, where they can survive and even multiply in protozoa. ${ }^{3}$ There are 42 species of Legionella and several different serotypes, but L. pneumophila serotype 1 is responsible for most lower respiratory tract infections. ${ }^{3,4}$ Hematogenous or lymphatic spread from the lungs to the liver, spleen, lymph nodes, myocardium, and kidneys may occur. ${ }^{3}$

Each year 1,200 to 1,800 cases of Legionella infection are reported to the Centers for Disease Control and Prevention, of which $20 \%$ to $25 \%$ are considered to be nosocomial. ${ }^{3,4}$ The number of cases reported annually may underestimate the magnitude, morbidity, and cost of nosocomial legionnaires' disease because of limited reporting, missed cases, and a lack of sensitive and specific methods of diagnosis., 3 Diagnostic tests for Legionella are also underused, and the commonly used urinary antigen test lacks sensitivity for many Legionella species and does not identify non-serotype 1 isolates of $L$. pneumophila. The increased use of the new fluoroquinolones and macrolides during the past decade may have decreased the reported incidence, risk, and mortality of Legionella pneumonia.

Many individuals believe that the water in hospitals is sterile. However, Legionella and other microorganisms may be isolated from institutional hot and cold water systems. The pathogenesis of nosocomial Legionella infections and the contamination of hospital water have been linked "like light and shadow" using different molecular typing methods. ${ }^{3-5}$ Recent surveys suggest that $12 \%$ to $75 \%$ of hospitals have water systems that are contaminated with Legionella ${ }^{4,6,7}$ Legionella are transmitted to humans via contaminated aerosols from cooling towers, showers, faucet aerators, nebulizers, humidifiers, or ice machines or by aspiration of contaminated water or pharyngeal contents; person-to-person transmission is uncommon. A patient's risk of acquiring Legionella pneumonia is related to the type and intensity of the exposure, advanced age, smoking, or a chronic underlying disease. ${ }^{3-5.7}$ Patients undergoing bone marrow or solid organ transplants and other immunosuppressed patients are at highest risk for infection.

With an aging patient population in U.S. hospitals that has more chronic disease and immunosuppression, measures should be taken to prevent nosocomial Legionella infections. What can be done? Healthcare facilities should formulate a strategy based on their patient population, facility design, resources, and available methods for control. ${ }^{3,4}$ Two schools of thought have emerged for hospitals with either no cases or a few, sporadic cases. Yu et al. suggest routine culturing of the water samples from the facility. ${ }^{8}$ If the cultures for Legionella are negative, then the risk of nosocomial legionellosis is low. If the cultures are positive, physicians and other professional staff should be notified, appropriate diagnostic testing for Legionella performed, surveillance increased, and water decontamination performed. 
The Centers for Disease Control and Prevention argues that culturing is expensive and that the correlation between water contamination and nosocomial Legionella infections is unpredictable. Centers for Disease Control and Prevention recommendations include a high index of suspicion for legionellosis, use of appropriate diagnostic tests for patients with suspected pneumonia, and, if one definite case or two possible cases of Legionella are identified, culturing and decontamination of water as outlined in the Centers for Disease Control and Prevention and Hospital Infection Control Practices Advisory Committee Guidelines for Prevention of Nosocomial Pneumonia. ${ }^{4}$ To date, water decontamination with hyperchlorination has been disappointing as a long-term solution, due to high expense, pipe corrosion, and difficulties maintaining the 2 to $4 \mathrm{ppm}$ of chlorine needed to sustain efficacy.,9 Furthermore, thermal eradication is tedious and labor intensive and contamination often recurs within a month., 3

Data on several methods of water decontamination are presented in this issue of Infection Control and Hospital Epidemiology that shed new light on some gray areas of Legionella prevention. ${ }^{9-12}$ Stout and Yu propose a scientific standard for evaluating water disinfection methods and provide valuable data on the long-term efficacy of disinfection with copper-silver ionization. ${ }^{9}$ Eradication of Legionella was associated with elimination of legionnaires' disease at 16 hospitals during a 5 -year period. All 16 hospitals had previous cases of nosocomial Legionella, and $75 \%$ of these had unsuccessfully used other methods of disinfection, including superheat and flush, ultraviolet light, and hyperchlorination. After installation of copper-silver ionization systems, $50 \%$ of the hospitals reported $0 \%$ positivity, and $43 \%$ were still culture negative 5 years later. The cost of the systems ranged from $\$ 6,000$ to $\$ 134,572$, with additional costs for routine maintenance and monitoring of the systems and the $\mathrm{pH}$ of the water. These data also suggest that control of legionnaires' disease may not require complete elimination of the organism.

Srinivasan et al. present data on a 17-month evaluation of a chlorine dioxide water treatment system to control Legionella in a hospital that housed surgical and oncology patients, including bone marrow transplant recipients. ${ }^{11}$ This is the first report of the safety and efficacy of this disinfection method in the United States, and the data confirm earlier reports from Europe. After disinfection was initiated, sites growing Legionella decreased from $41 \%$ to $4 \%(P=$ .001 ), and only Legionella anisa was cultured. No cases of nosocomial Legionella were identified in the buildings where chlorine dioxide was used. Chlorine dioxide and chlorite levels were maintained below the Environmental Protection Agency limits for potable water by carbon filtration, and no evidence of pipe corrosion was noted. The study was limited by the short duration of the evaluation and the lack of data on the costs of implementing and maintaining the system.

Hall et al. present interesting data on the use of ultraviolet light to prevent Legionella in a new hospital, with Legionella having contaminated the water of the old hospi- tal across the street. ${ }^{12}$ During a 13-year period of intensive surveillance, none of the 930 surveillance cultures of hos-. pital tap water was positive and only one case of nosocomial $L$. pneumophila was identified. This occurred in a transplant patient who probably acquired the organism at another hospital prior to transfer. Ultraviolet light has been shown to kill Legionella in vitro, and has been used in combination with other disinfection methods. As pointed out by the authors, because ultraviolet light provides no residual protection in the pipes located downstream, which may contain biofilm in which Legionella can live and multiply, perhaps the aim should be to prevent contamination. Ultraviolet light was initially used at this institution to control an outbreak of $L$. micdade $i$ from 1978 to $1982 .{ }^{13}$ New water pipes and a ultraviolet lamp were installed into the hot and cold water systems leading to rooms occupied by renal transplant patients. After installation, cultures were negative and the outbreak subsided. These data on ultraviolet light may be particularly helpful for planning Legionella control systems in new hospitals, and for any remodeling needed to protect immunosuppressed patients.

Water supplies disinfected with monochloramine by municipal plants have been previously noted to have lower rates of Legionella contamination. ${ }^{14}$ In this issue of Infection Control and Hospital Epidemiology, Heffelfinger et al. present intriguing data to support these findings, using a survey of 459 members of the Society for Healthcare Epidemiology of America (SHEA) representing 166 hospitals. ${ }^{10}$ Of the 166 hospitals, $33(20 \%)$ had reported one or more episodes of nosocomial legionnaires' disease and 23 (14\%) had reported a previous outbreak. Compared with hospitals with no cases, those with nosocomial Legionella cases had a significantly higher $(P=.03)$ census, conducted surveillance, had more acute care beds, and had a transplant service. After adjustment for these variables, hospitals supplied with drinking water disinfected with monochloramine by municipal plants were significantly less likely than other hospitals to have sporadic cases or outbreaks of nosocomial Legionella pneumonia (odds ratio, 0.20; 95\% confidence interval, 0.07 to 0.56 ). Thus, the use of water treated with monochloramine by municipal plants may be a cost-effective method for public health departments and hospitals to adopt in the battle against nosocomial and community-acquired legionnaires' disease.

Dr. William Osler's warning at the beginning of the 20 th century to "Remember how much you don't know" remains true today. However, if Dr. Osler were alive today, he undoubtedly would be delighted with the enormous scientific progress we have made and, therefore, might be more concerned over what we do know and haven't applied. In a healthcare system stressed by financial burdens, limited resources, and formidable challenges, there is a need for greater emphasis on disease prevention. Nosocomial infections such as Legionella are an important target for the 21st century. Interest in preventing contamination of water treatment systems as a source of opportunistic infection will continue to grow. Data contained in this issue of Infection Control and Hospital Epidemiology 
shed new light on several gray areas surrounding prevention strategies for Legionella, but additional long-term efficacy and toxicity data and methods of implementing effective prevention programs will be needed to declare victory. In addition, national or minimal standards for hospital water systems and regulation should be discussed. One solution to this prevention conundrum is contained in an old African adage: "The best time to plant a tree is 20 years ago, the second best time is now."

\section{REFERENCES}

1. Fraser D, Tsai T, Orenstein W, et al. Legionnaires' disease: description of an epidemic of pneumonia. N Engl J Med 1977;297:1189-1197.

2. McDade J, Shepard C, Fraser D. Legionnaires' disease: isolation of a bacterium and demonstration of its role in other respiratory disease. $N$ Engl J Med 1977;297:1197-1203.

3. Stout J, Yu V. Nosocomial Legionella infection. In: Mayhall C, ed. Hospital Epidemiology and Infection Control. Philadelphia: Lippincott, Williams and Wilkins; 1999:453-465.

4. Centers for Disease Control and Prevention, Hospital Infection Control Practices Advisory Committee. Guidelines for prevention of nosocomial pneumonia. MMWR 1997:46(RR1):1-79.

5. Kirby B, Snyder K, Meyer R, et al. Legionnaires' disease: report of sixtyfive nosocomially acquired cases and review of the literature. Medicine
1980;59:188-205.

6. Yu V. Resolving the controversy on environmental cultures for Legionella: a modest proposal. Infect Control Hosp Epidemiol 1998;19:893-897.

7. Stout JE, Yu VL. Legionellosis. N Engl J Med 1997;337:682-687.

8. Yu V, Beam TR, Lumish RM, et al. Routine culturing for Legionella in the hospital environment may be a good idea: a three-hospital prospective study. Am J Med Sci 1987;294:97-99.

9. Stout JE, Yu VL. Experiences of the first 16 hospitals using cooper-silver ionization for Legionella control: implications for the evaluation of other disinfection modalities. Infect Control Hosp Epidemiol 2003;24:563568 .

10. Heffelfinger JD, Kool JL, Fridkin S, et al. Risk of hospital-acquired legionnaires' disease in cities using monochloramine versus other water disinfectants. Infect Control Hosp Epidemiol 2003;24:569-574.

11. Srinivasan A, Bova G, Ross T, et al. A 17-month evaluation of a chlorine dioxide water treatment system to control Legionella species in a hospital water supply. Infect Control Hosp Epidemiol 2003:24:575-579.

12. Hall KK, Giannetta ET, Getchell-White SI, Durbin LJ, Farr BM. Ultraviolet light disinfection of hospital water for preventing nosocomial Legionella infection: a 13 year follow-up. Infect Control Hosp Epidemiol 2003;24:580-583.

13. Farr B, Gratz J, Tartaglino J, et al. Evaluation of ultraviolet light for disinfection of hospital water contaminated with Legionella. Lancet 1999;1:669-671.

14. Kool JL, Carpenter JC, Fields BS. Effects of monochloramine disinfection of municipal drinking water on risk of nosocomial legionnaires' disease. Lancet 1999;353:272-277. 